\title{
EXPERIMENTAL INVESTIGATION OF TWO-DEGREE-OF-FREEDOM VIV OF CIRCULAR CYLINDER WITH LOW EQUIVALENT MASS AND VARIABLE NATURAL FREQUENCY RATIO
}

\author{
Narakorn Srinil ${ }^{\star}$, Hossein Zanganeh, Alexander Day \\ Department of Naval Architecture and Marine Engineering, University of Strathclyde \\ Glasgow, Scotland, UK, "corresponding author: narakorn.srinil@strath.ac.uk
}

\begin{abstract}
This paper presents an experimental investigation and validation of numerical prediction model for a 2-DOF VIV of a flexibly mounted circular cylinder by also accounting for the effect of geometrically nonlinear displacement coupling. A mechanical spring-cylinder system, achieving a low equivalent mass ratio in both in-line and cross-flow directions, is tested in a water towing tank and subject to a uniform steady flow in a sub-critical Reynolds number range of about 2000-50000. A generalized numerical model is based on double Duffing-van der Pol (structure-wake) oscillators which can capture the structural geometrical coupling and fluid-structure interaction effects through system cubic and quadratic nonlinearities. Experimental results are compared with numerical predictions in terms of response amplitudes, lock-in ranges and timevarying trajectories of cross-flow/in-line motions. Some good qualitative and quantitative agreements are found which encourage the use of the proposed numerical model subject to calibration and tuning of empirical coefficients. Various features of figure-of-eight orbital motions due to dual resonances are observed experimentally as well as numerically, depending on the natural frequency ratio of the oscillating cylinder.
\end{abstract}

\section{INTRODUCTION}

Vortex-induced vibration (VIV) has received a very high interest over the past decades due to the variety of nonlinear phenomena governed by the fluid mechanics, structural dynamics and fluid-structure interactions. In many ocean and offshore engineering applications, VIV continues to be of great concern in the context of fatigue analysis, design and operation of deep water structures exposed to ocean currents. From a theoretical and practical viewpoint, both experimental tests and numerical prediction models - capable of capturing VIV occurrences and behaviors in a wide range of both the hydrodynamics and the structural parameters - are important. However, in spite of many published studies, the vast majority of the research literature has focused on one-dimensional crossflow VIV of a circular cylinder for which the transverse response is typically observed to be the largest (Bearman, 2011; Sarpkaya, 2004; Williamson and Govardhan, 2004), and on the related semi-empirical modelling of a 1-degree-of-freedom (DOF) cross-flow-only VIV (Gabbai and Benaroya, 2005). Experimental studies and comparisons with numerical prediction results for two-dimensional in-line $(\mathrm{X})$ and crossflow (Y) or 2-DOF VIV are still rather limited and therefore needed to be further addressed comprehensively.

In this study, new experimental VIV results of a 2-DOF circular cylinder with equivalent mass ratio in both $\mathrm{X}-\mathrm{Y}$ directions $\left(m^{*}{ }_{x}=m^{*}{ }_{y}\right)$ and variable in-line-to-cross-flow natural frequency ratio $\left(f^{*}=f_{n x} / f_{n y}\right)$ are presented and compared with the associated numerical outcomes predicted by new nonlinear structure-wake oscillators (Srinil and Zanganeh, 2012). Some insightful VIV aspects are also discussed in the light of other published experimental results with variable $f^{*}$ but $m_{x}^{*} \neq m_{y}{ }_{y}$ (e.g. Dahl et al., 2006). Note that the condition of $m^{*}{ }_{x}=m^{*}{ }_{y}$ is more relevant in practice than that of $m^{*} \neq m^{*}$ to real cylindrical offshore structures including risers, mooring cables and pipelines. The $f^{*}$ variation is also of practical relevance because such a distributed-parameter system contains an infinite number of natural frequencies in different directions entailing various $f^{*}$ (Srinil and Rega, 2007; Srinil et al., 2007; 2009). These can result in different lock-in or resonant conditions with the vortex shedding frequencies of the fluctuating lift and drag forces. As the drag oscillation has double the frequency of the lift oscillation, a perfect two-dimensional resonance case might occur when $f^{*}=2$. This circumstance could potentially lead to a 
large-amplitude response for a system with low mass and damping parameter.

Recent experimental studies have highlighted some interesting features of 2-DOF VIV of circular cylinders and meaningful contributions from the in-line VIV to the overall dynamics, depending on several control parameters. In general, the freedom of the system to oscillate in the in-line direction can cause an increase of the cross-flow response amplitude and widen the lock-in range (Moe and Wu, 1990; Sarpkaya, 1995); it has been suggested that these effects may result from an enhanced correlation of the transverse force along the cylinder span (Moe and $\mathrm{Wu}, 1990)$. With respect to the flow field visualization, a new $2 \mathrm{~T}$ (two of vortex triplets) wake mode has been observed for the cylinder with significant combined $\mathrm{X}-\mathrm{Y}$ motion (Williamson and Jauvtis, 2004) in addition to the typical $2 \mathrm{~S}$ (two single vortices) and $2 \mathrm{P}$ (two vortex pairs) modes defined in the Y-only cylinder motion case (Khalak and Williamson, 1999).

With $m^{*}{ }_{x}=m^{*}$ and $f^{*}=1$, Jauvtis and Williamson (2004) showed that there is a slight influence on the cross-flow response of the cylinder with $m^{*}>6$ when comparing the results obtained between 1- and 2-DOF models. When $m^{*}<6$, there is a super-upper branch in the cross-flow response with the peak amplitude $A_{y} / D=1.5$ coexisting with the in-line response with the peak amplitude $A_{x} / D=0.3$, along with response jump and hysteresis phenomena. Similar nonlinear responses and ranges of maximum $A_{x} / D$ and $A_{y} / D$ have been experimentally reported by Stappenbel et al. (2007) and Belvins and Coughran (2009). A two-dimensional lock-in range is found to be mainly influenced by the variation of the mass ratio (Stappenbelt et al., 2007). However, both mass (Stappenbelt et al., 2007) and damping (Blevins and Coughran, 2009) parameters can influence on 2-DOF peak amplitudes.

With $m^{*}{ }_{x} \neq m^{*}$ and $f^{*} \neq 1$, different qualitative and quantitative features of 2-DOF VIV responses appear. In particular, a two-peak cross-flow response has been noticed by Sarpkaya (1995) and Dahl et al. (2006) with $f^{*}=2$ and 1.9, respectively. Dahl et al. (2010) further highlighted various figure-of-eight patterns in different subcritical and supercritical Reynolds number $(\mathrm{Re})$ ranges $\left(1.5 \times 10^{4}<\mathrm{Re}<6 \times 10^{4}\right.$ and $\left.3.2 \times 10^{5}<\operatorname{Re}<7.1 \times 10^{5}\right)$ and described a figure-eight occurrence as a representation of "dual resonance". Under this dual resonance, the frequencies of the unsteady drag and lift forces are resonantly tuned with $f_{x}$ and $f_{y}$, respectively, such that $f_{x} / f_{y} \neq$ 2. In addition, a large third harmonic component of the lift force was observed although the main cross-flow response was primarily associated with the first-harmonic lift force. This is in agreement with a lab experiment of a flexible cylinder by Trim et al. (2005).

In spite of the above-mentioned studies, the most practical case of $m^{*}{ }_{x}=m^{*}{ }_{y}$ and variable $f *$ has not been thoroughly investigated. More experimental and numerical investigations in a higher Re range are still needed along with the improvement of relevant prediction models.
The main objectives of the present study are to (i) experimentally investigate 2-DOF VIV of a flexibly mounted circular cylinder with $m^{*} x_{x}=m_{y}^{*}$ and variable $f^{*}$; and (ii) compare the obtained experimental results with numerical prediction outcomes in order to justify the newly-proposed coupled oscillators (Srinil and Zanganeh, 2012) with a proper choice of system coefficients. To calibrate model empirical coefficients, particular attention is placed on the determination of cylinder maximum attainable $A_{x} / D$ and $A_{y} / D$, associated lockin ranges (both the onset and the end of synchronization), and two-dimensional orbital X-Y motions, by comparing the cases of $f^{*} \neq 1$ and $f^{*}=1$. These analysis outcomes based on a 2-DOF rigid cylinder could be practically useful in the improvement of VIV prediction tools and design guidelines for cross-flow/inline VIV of flexible cylinders with multi DOF and various $f^{*}$, as conducted, for instance, by Srinil $(2010,2011)$ for cross-flowonly VIV cases.
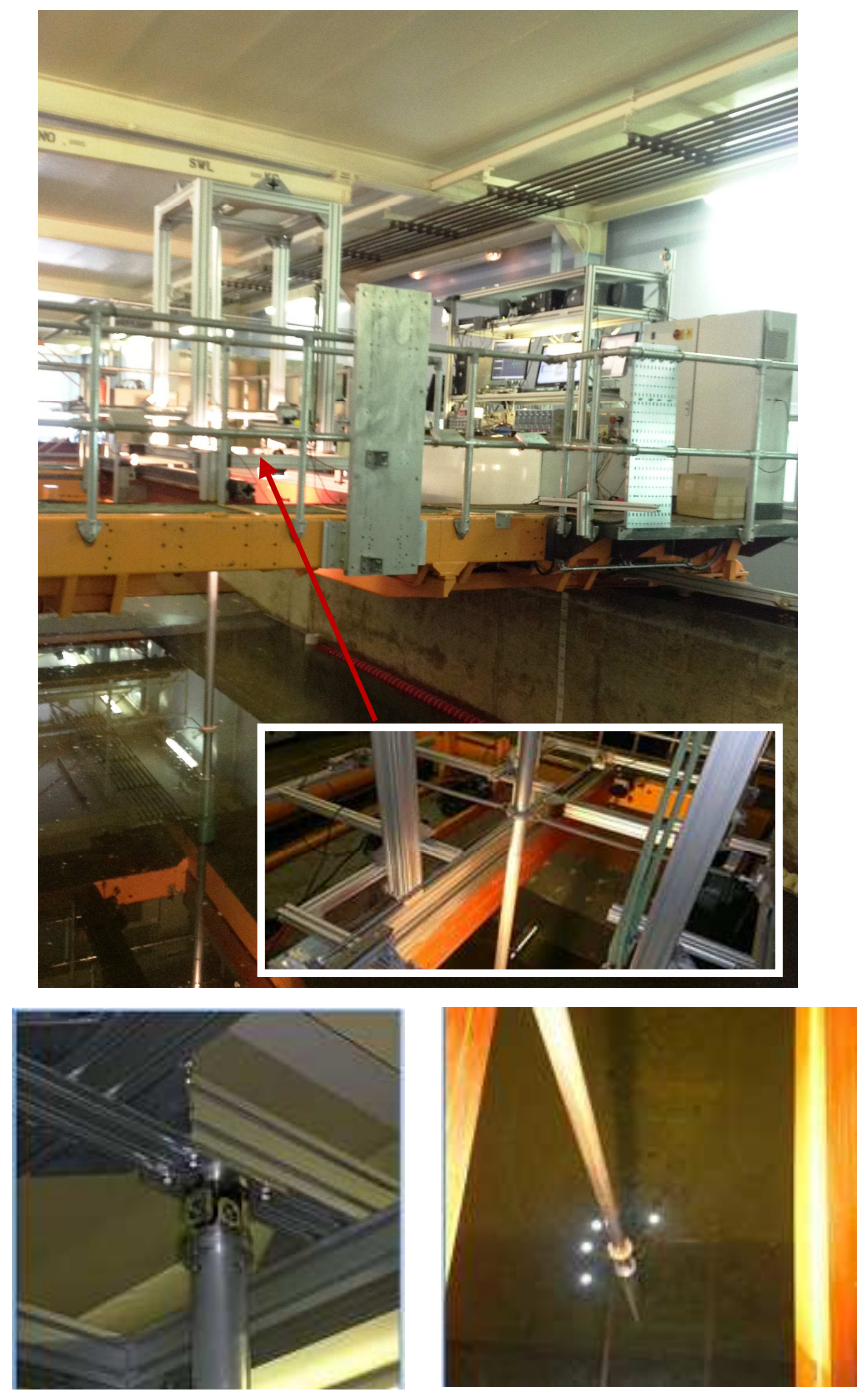

Figure 1 Experimental model and arrangement 


\section{EXPERIMENTAL MODEL AND PROCEDURE}

A new experimental test rig for the study of 2-DOF VIV of a flexibly mounted, smooth and rigid circular cylinder subject to a uniform steady flow has been developed for use in the towing tank at the Kelvin Hydrodynamics Laboratory (KHL) of the University of Strathclyde, Glasgow, UK. The KHL tank has dimensions of $76 \mathrm{~m}$ long by $4.57 \mathrm{~m}$ wide; water depth can be varied from $0.5-2.3 \mathrm{~m}$. The tank is equipped with a selfpropelled towing carriage on which the experimental apparatus can be firmly installed. Figure 1 displays the experimental setup. The test cylinder is mounted vertically and connected at its upper end to a long aluminum pendulum with total length of about $4.1 \mathrm{~m}$. The pendulum is attached to the supporting framework via a high-precision universal joint at the top of the frames. The test cylinder adopted in the present study is made of thick-walled cast nylon tube, having an outer diameter $(D)$ of $114 \mathrm{~mm}$ and a fully submerged length $(L)$ of $1.037 \mathrm{~m}$. The lower end of the cylinder is located $50 \mathrm{~mm}$ from the bottom of the tank, and the upper end is located $50 \mathrm{~mm}$ beneath the static free surface. It should be noted that the pendulum effect on the uniformity of the local flow field is believed to be insignificant since the maximum roll and pitch angles of the cylinder about the vertical axis were found to be only about 2 degrees in all tests. The blockage is about $2.5 \%$ and the aspect ratio $(L / D)$ of the cylinder is about 9.

The mechanical system is restrained to allow the cylinder to oscillate freely with arbitrary amplitudes in both in-line (X) and cross-flow $(\mathrm{Y})$ directions by using two pairs of coil springs rearranged perpendicularly in the horizontal $\mathrm{X}-\mathrm{Y}$ plane. This assembly creates a geometrically nonlinear stiffness and coupling of cross-flow/in-line displacements. These non-linear effects are also accounted for in the numerical prediction model. Measurement of cylinder motions was carried out using a Qualisys infrared optical tracking camera system with a fixed sampling frequency of $137 \mathrm{~Hz}$. When the cylinder is towed, the mean drag causes mean in-line displacement of the cylinder in the fluid flow direction. In order that the mean position of the cylinder for the measurements is vertical in the $\mathrm{X}$ direction, this displacement was adjusted by pre-tensioning the in-line spring such that the cylinder mean position, as measured using the Qualisys system, remains nearly vertical during the test.

Our attention in the present study is placed on the cylinder model with varying ratios between in-line $\left(f_{n x}\right)$ and cross-flow $\left(f_{n y}\right)$ natural frequencies in water $\left(f^{*}=f_{n x} / f_{n y}\right)$; this was achieved in practice by using springs with differing stiffness. The reported experimental cross-flow $\left(A_{y} / D\right)$ and in-line $\left(A_{x} / D\right)$ amplitudes normalized by the cylinder diameter are referred to as the maximum displacements at the bottom tip of the cylinder. Based on a free decay test in air, the experimental apparatus with and without the cylinder-spring system was found to be lightly damped at around $0.5 \%$ and $0.2 \%$ of the critical damping, respectively. A series of free decay tests in calm water were performed to identify the cylinder natural frequencies in water $\left(f_{n x}, f_{n y}\right)$ and the associated damping ratios $\left(\xi_{x}, \xi_{y}\right)$ in both
$\mathrm{X}$ and $\mathrm{Y}$ directions. The averaged $\xi_{x}$ and $\xi_{y}$ values have been evaluated by subtracting the fluid damping component from the total damping of the system (Sumer and Fredsoe, 2006).

Table 1 summarizes a test matrix of 3 datasets in which the mass ratio $m^{*}{ }_{x}=m^{*}{ }_{y}=1.4$ of the cylinder is considered. Due to the amplitude-dependence nature of the structural and fluidadded damping in water, variable $\xi_{x}$ and $\xi_{y}$ values (between 1-5 $\%)$ are reported. The combined mass-damping $m^{*} \xi$ values are reasonably low enough to encourage significant cross-flow and in-line displacements. Three different $f^{*}=1.01,1.29$ and 1.85 were performed. In all datasets, the reduced flow velocity $V_{r}$ range in which $V_{r}=V / f_{n y} D$ was about $0<V_{r}<20$, corresponding to $2 \times 10^{3}<\mathrm{Re}<5 \times 10^{4}$ of the sub-critical flows and the flow speed $V$ of $0.02-0.6 \mathrm{~m} / \mathrm{s}$. This considered range encompassed a $V_{r}$ value at which the peak amplitude occurred. Some tests were repeated in the neighborhood of peaks and response jumps.

Table 1 Experimental datasets and parameters

\begin{tabular}{|c|c|c|c|}
\hline Dataset & $\xi_{y}(\%)$ & $\xi_{x}(\%)$ & $f^{*}$ \\
\hline 1 & 1.0 & 4.7 & 1.01 \\
\hline 2 & 1.5 & 1.0 & 1.29 \\
\hline 3 & 1.8 & 1.2 & 1.85 \\
\hline
\end{tabular}

\section{NUMERICAL PREDICTION MODEL}

The capability to reasonably model and accurately predict the coupled cross-flow/in-line VIV structural response excited by the unsteady flow field has been a major challenge to modelers and offshore engineers for many years although some prediction tools are currently available in the industry.

A schematic model of the cylinder restrained by two pairs of springs to oscillate in $\mathrm{X}$ and $\mathrm{Y}$ directions is displayed in Fig. 2a. The key aspect in the formulation of system equations of motions is to capture the quadratic relationship between in-line and cross-flow displacements (Vandiver and Jong, 1987). Following Wang et al. (2003), the two-directional unsteady fluid forces can be exerted on the oscillating cylinder as opposed to the stationary one, by also accounting for the relative velocities between the incoming flow and the cylinder in-line motion. As a result, the sectional lift $\left(F_{L}\right)$ and drag $\left(F_{D}\right)$ forces coincide with an arbitrary plane making up an angle of $\theta$ with respect to the $\mathrm{Y}$ and $\mathrm{X}$ axes, respectively.

Two cases can be realized depending on whether $\theta$ is counterclockwise (Fig. 2b) or clockwise (Fig. 2d). From our numerical simulation experience, it has been discovered that such $\theta$ direction plays a key role in the ensuing phase difference between cross-flow and in-line oscillations and, in turn, the figure-of-eight appearing shape. In general, the orbital plot exhibits a figure-eight trajectory with tips pointing upstream with a counterclockwise $\theta$ model (e.g. Fig. 2c) or downstream with a clockwise $\theta$ model (e.g. Fig. 2e). As both cases have been experimentally observed in the literature, they are herein accounted for in the generalized model formulation. 

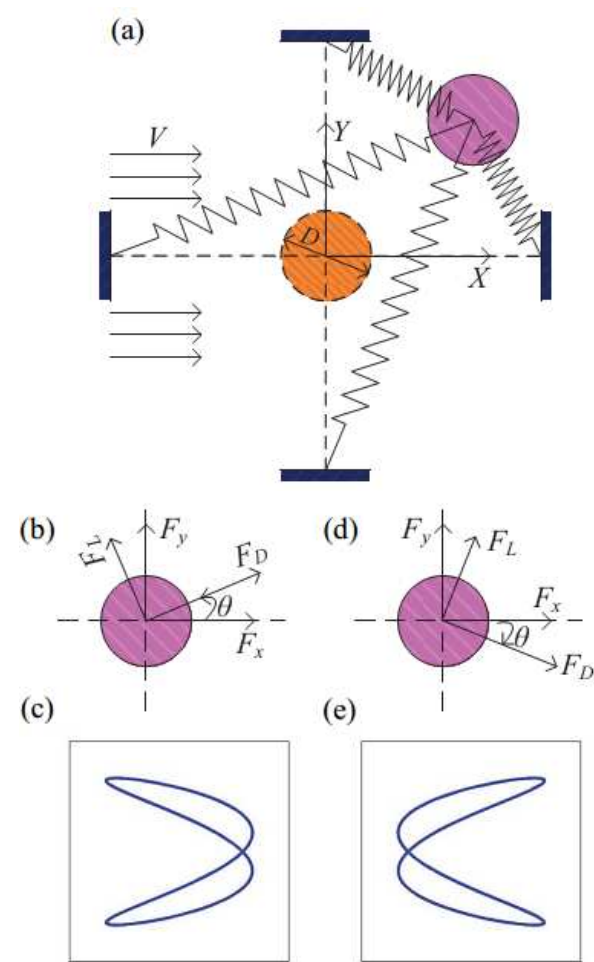

Figure 2 Numerical model

By assuming a small $\theta$, the unsteady hydrodynamic forces $F_{x}$ and $F_{y}$ may be simplified after resolving $F_{L}$ and $F_{D}$ into the $\mathrm{X}$ and $\mathrm{Y}$ directions as

$$
\begin{aligned}
& F_{x}=F_{D} \cos \theta \mp F_{L} \sin \theta \approx F_{D} \mp F_{L} \dot{\tilde{Y}} / V, \\
& F_{y}=F_{L} \cos \theta \pm F_{D} \sin \theta \approx F_{L} \pm F_{D} \dot{\tilde{Y}} / V,
\end{aligned}
$$

where $\dot{\tilde{Y}}$ is the dimensional transverse displacement, a dot denotes differentiation with respect to the dimensional time $T$, $\rho$ is the fluid density, $C_{D}$ and $C_{L}$ are the time-varying drag and lift coefficients, the minus (positive) and positive (minus) sign in Eq. (1) (Eq. 2) correspond to the case of counterclockwise and clockwise $\theta$, respectively.

By assigning the fluid vortex variables as $p=2 C_{D} / C_{D O}$ and $q=2 C_{L} / C_{L O}$ (Facchinetti et al., 2004) in which $C_{D O}$ and $C_{L O}$ are the associated drag and lift coefficients of a stationary cylinder (assumed as $C_{D O}=0.2$ (Currie and Turnbull, 1987) and $C_{L 0}=0.3$ (Blevins, 1990)), the time variation of $p$ and $q$ may be assumed to follow the self-excitation and -limiting mechanism of the van der Pol wake oscillators. By introducing the dimensionless time $t=\omega_{n y} T$ and normalizing the displacements with respect to $D$, the nonlinearly coupled equations describing the in-line $(x)$ and cross-flow $(y)$ oscillations of the cylinder subject to the fluctuating fluid force components $(p, q)$ may be expressed in dimensionless form as (Srinil and Zanganeh, 2012)

$$
\begin{aligned}
& \ddot{x}+\lambda_{x} \dot{x}+f *^{2}\left(x+\alpha_{x} x^{3}+\beta_{x} x y^{2}\right)=M_{D} \Omega^{2} p \mp 2 \pi M_{L} \Omega^{2}\left(q \dot{y} / V_{r}\right), \\
& \ddot{p}+2 \varepsilon_{x} \Omega\left(p^{2}-1\right) \dot{p}+4 \Omega^{2} p=\Lambda_{x} \ddot{x}
\end{aligned}
$$

$$
\begin{aligned}
& \ddot{y}+\lambda_{y} \dot{y}+y+\alpha_{y} y^{3}+\beta_{y} y x^{2}=M_{L} \Omega^{2} q \pm 2 \pi M_{D} \Omega^{2}\left(p \dot{y} / V_{r}\right), \\
& \ddot{q}+\varepsilon_{y} \Omega\left(q^{2}-1\right) \dot{q}+\Omega^{2} q=\Lambda_{y} \ddot{y}
\end{aligned}
$$

in which

$$
\begin{aligned}
& M_{D}=C_{D 0} / 16 \pi^{2} \mathrm{St}^{2} \mu, M_{L}=C_{L 0} / 16 \pi^{2} \mathrm{St}^{2} \mu, \\
& \mu=\left(m_{s}+m_{f}\right) / \rho D^{2}, \\
& \lambda_{x}=2 \xi_{x} f^{*}+\gamma \Omega / \mu, \lambda_{y}=2 \xi_{y}+\gamma \Omega / \mu,
\end{aligned}
$$

$\Omega=\mathrm{St} V_{r}, m_{f}=\pi \rho D^{2} C_{M} / 4, m_{s}$ is the cylinder mass, $m_{f}$ the fluid added mass, $C_{M}$ the added mass coefficient assumed to be unity for a circular cylinder (Blevins, 1990), St the Strouhal number, $\gamma$ the stall parameter which is directly related to the sectional mean drag coefficient and assumed to be a constant equal to 0.8 (Facchinetti et al., 2004), and co-subscripts $x$ and $y$ identify properties in these directions. Note that the mass ratio definition in the literature is variable but the widely recognized one with $m^{*}=4 \mu / \pi-C_{M}$ is herein considered (Williamson and Govardhan, 2004).

In contrast to typical VIV models which consider a linear structural oscillator to describe the cylinder displacement (Gabbai and Benaroya, 2005), Eqs. (3) and (5) account for the effect of geometric nonlinearities (i.e. nonlinear stiffness or restoring force) of the oscillating cylinder. These equations are so-called Duffing-type oscillators (Nayfeh, 1993). Cubic nonlinear terms capture the effect of nonlinear stretching $\left(x^{3}, y^{3}\right)$ and physical cross-flow/in-line displacement coupling $\left(x y^{2}, x^{2} y\right)$, depending on the geometrical parameters $\left(\alpha_{x}, \alpha_{y}, \beta_{x}, \beta_{y}\right)$. Quadratic nonlinear terms have been found to be responsible for the figure-of-eight appearance associated with a 2:1 resonance condition (Srinil and Zanganeh, 2012). The coupling and interaction between the fluid and the structure is captured through all linear and nonlinear terms in the right-hand side of Eqs. (3)-(6). It is also worth remarking that, in Eqs. (3) and (5), the maximum cross-flow/in-line amplitudes are unaffected by the choice of $\theta$ since the associated velocities are trivial.

The analysis and prediction of coupled cross-flow/in-line VIV depend on a number of empirical coefficients $\left(\varepsilon_{x}, \varepsilon_{y}, \Lambda_{x}\right.$, $\left.\Lambda_{y}\right)$ and geometrical parameters $\left(\alpha_{x}, \alpha_{y}, \beta_{x}, \beta_{y}\right)$. Based on calibration with experimental results (Stappenbelt et al., 2007) with varying $m^{*}$ and $f^{*}=1$, it may be assumed that

$\varepsilon_{y}=0.00234 e^{\left(0.228 m_{y}^{*}\right)}$

To reduce the time-consuming task involving the tuning of individual model coefficients, $\varepsilon_{x}=0.3, \Lambda_{x}=\Lambda_{y}=15$, and $\alpha_{x}=$ $\alpha_{y}=\beta_{x}=\beta_{y}=0.7$ are initially assumed in all $f^{*}$ cases, unless stated otherwise.

Eqs. (3)-(6) are nonlinearly coupled and numerically solved by using a fourth-order Runge-Kutta scheme with an adaptive time step enabling solution convergence and stability, and with 
assigned initial conditions at $t=0$ of $x=y=0, p=q=2$ and zero velocities.

Some of the nonlinear terms in Eqs. (3) and (5) entail the static drifts (Zanganeh and Srinil, 2012) which are, however, disregarded from numerical simulations as attention is placed on the oscillating components caused by VIV. In all simulation cases, $V_{r}$ is increasingly varied in steps of 0.1 .
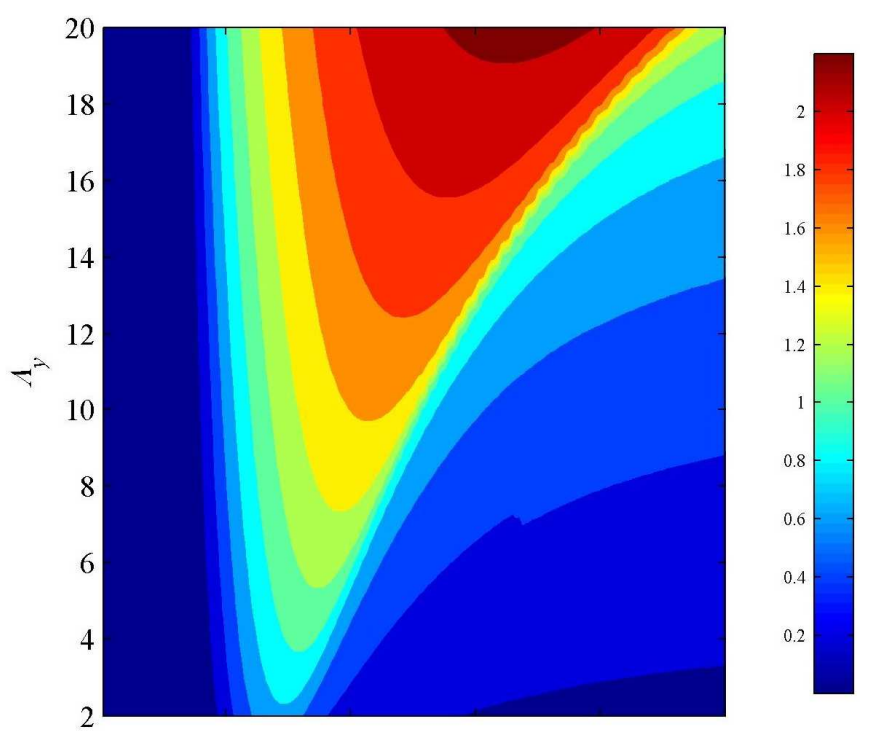

(a) $A_{y} D$
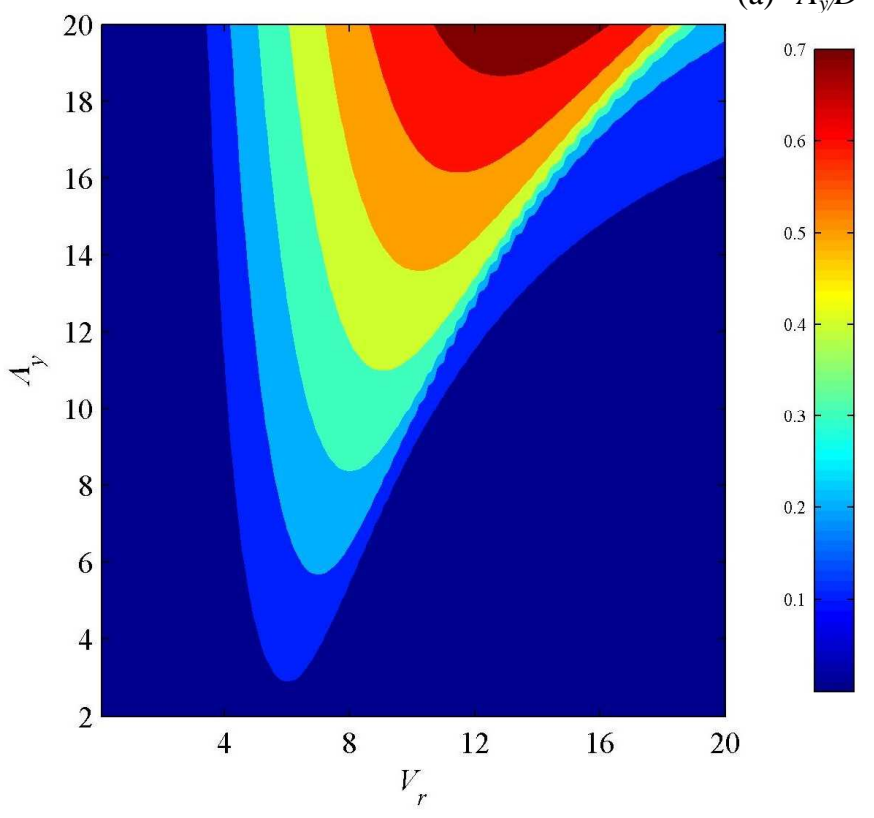

(b) $A_{x /} D$

Figure 3 Effect of varying $\Lambda_{y}$ on cross-flow and in-line amplitude responses

\section{SENSITIVITY STUDY ON EMPIRICAL COEFFICIENTS}

It is of practical importance to carry out a sensitivity study on the numerical model in order to understand the influence of varying parameters on the 2-DOF VIV predictions with variable $f^{*}$ cases. To also capture possible qualitative and quantitative changes, the sensitivity analysis should be performed with respect to the parameters related to the greater $y$ response (Srinil and Zanganeh, 2012).
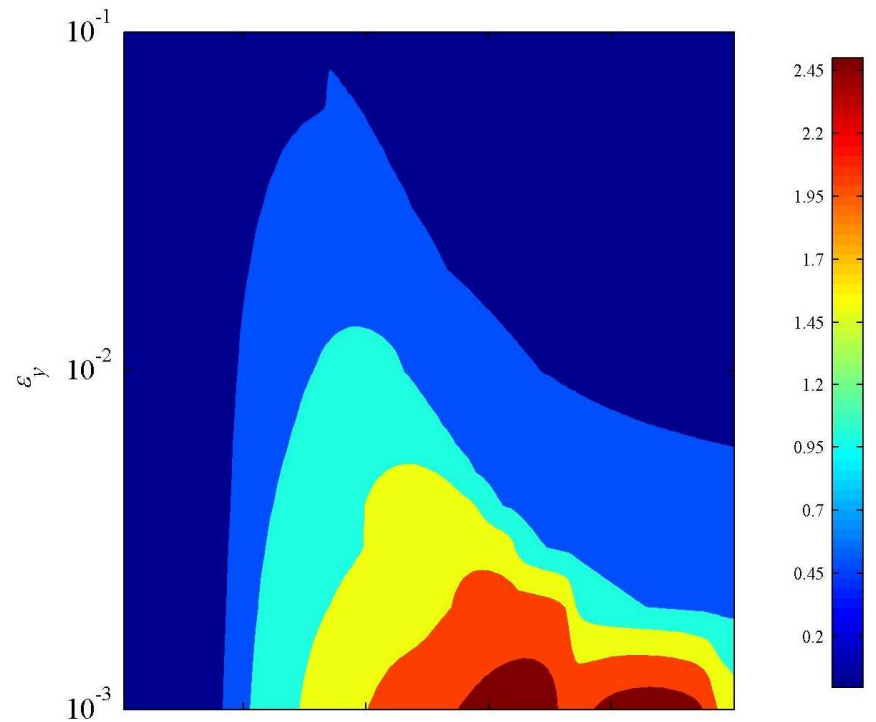

(a) $A_{y} D$
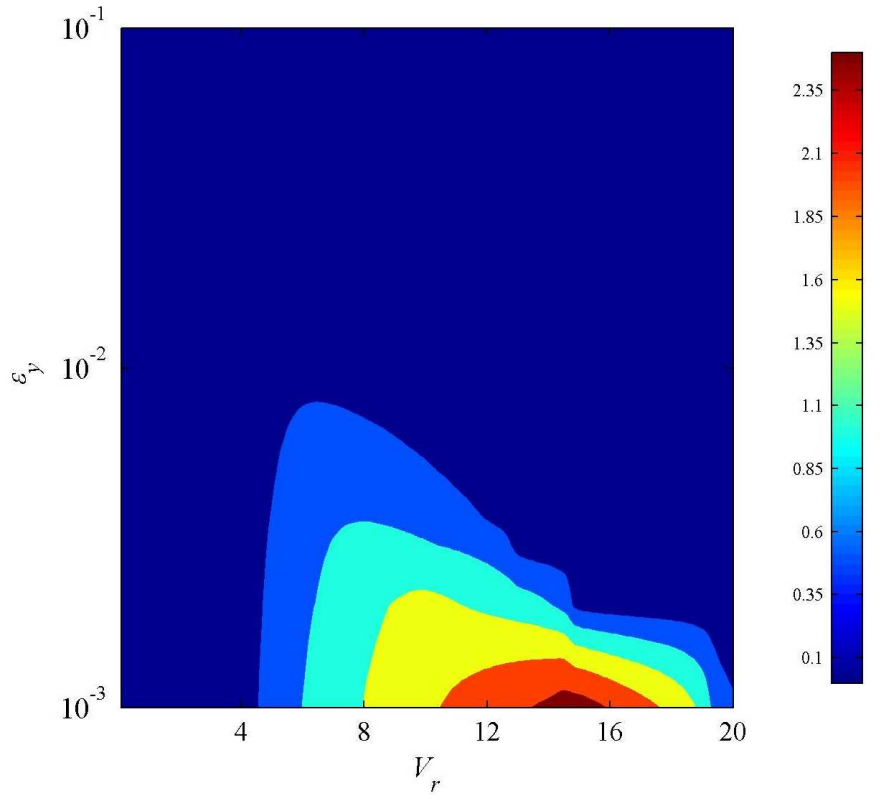

(b) $A_{x} D$

Figure 4 Effect of varying $\varepsilon_{y}$ on cross-flow and in-line amplitude responses 

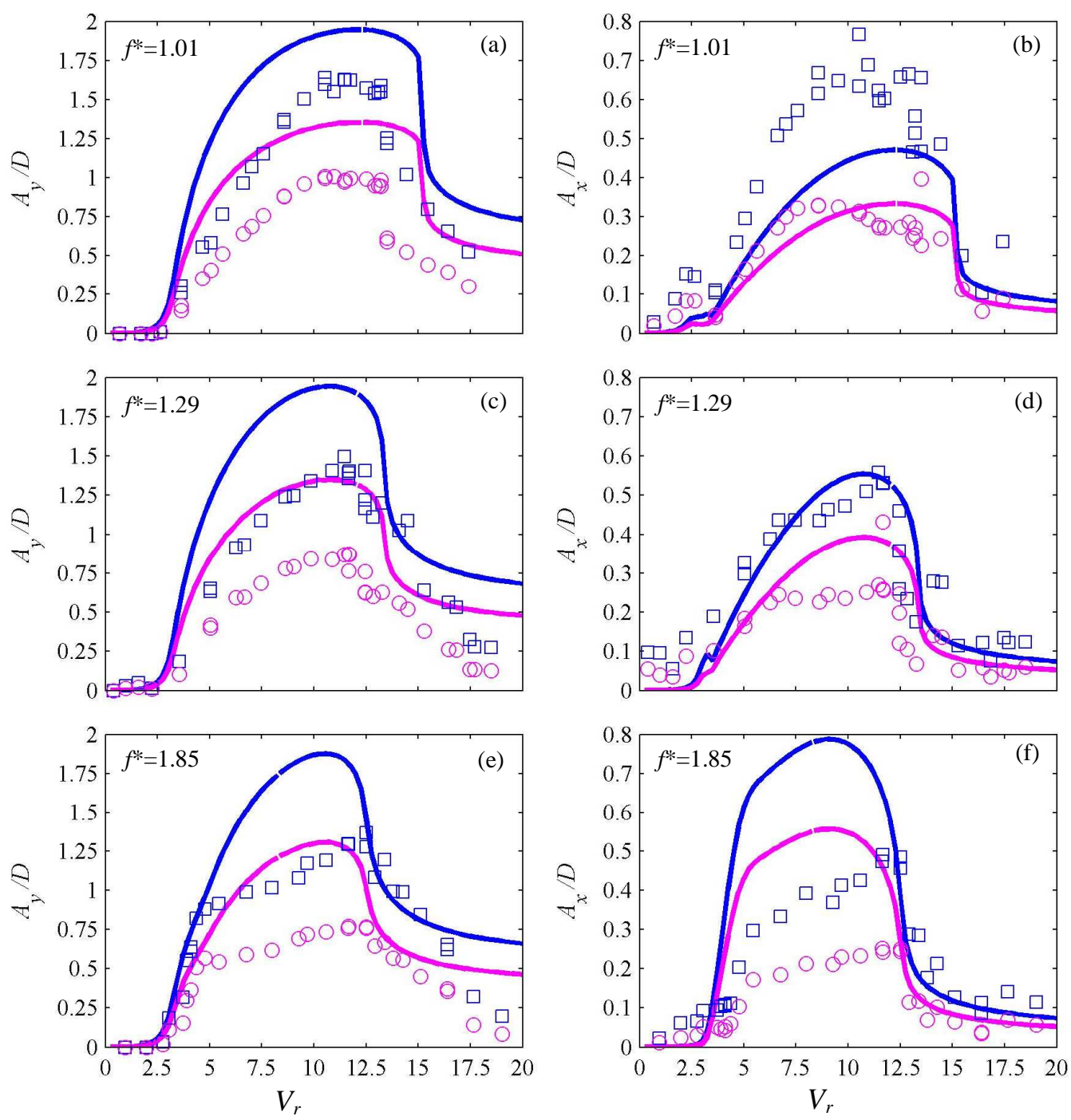

Figure 5 Cross-flow and in-line amplitudes of 3 datasets: blue (pink) lines denote numerically-predicted maximum (RMS) values; squares (circles) denote experimentally-obtained maximum (RMS) values

By ways of examples, the empirical coefficient $\Lambda_{y}$ or $\varepsilon_{y}$ is varied in the numerical simulations with $f^{*}=1.3$ or 2 , respectively. In each case, $\xi_{x}=1.6 \%$ and $\xi_{y}=1 \%$ are assigned. Contour plots of $A_{y} / D$ and $A_{x} / D$ are displayed in Figs. 3 and 4 in the varying $\Lambda_{y}$ and $\varepsilon_{y}$ cases, respectively.

It is seen in Fig. $3\left(f^{*}=1.3\right)$ that both $A_{y} / D$ and $A_{x} / D$ responses - as well as the associated lock-in ranges - increase as $\Lambda_{y}$ increases (from 2 to 20), with the associated peaks locating at higher $V_{r}$ values (from 5 to 12 ). As a result, the bentto-right nonlinear dynamic responses with high values of $\Lambda_{y}$ are influenced and controlled by the system cubic nonlinearities. The maximum attainable $A_{y} / D$ and $A_{x} / D$ are about 2.2 and 0.7 , respectively. Specifying low values of $\Lambda_{y}(<3)$ can lead to the purely cross-flow VIV response with negligible in-line VIV effect. These results highlight both quantitative and qualitative change when varying $\Lambda_{y}$.
On the contrary, results shown in Fig. $4\left(f^{*}=2\right)$ reveal how both $A_{y} / D$ and $A_{x} / D$ responses - as well as the associated lock-in ranges - increase as $\varepsilon_{y}$ decreases (from $10^{-1}$ to $10^{-3}$ ). Interestingly, for very low values of $\varepsilon_{y}$, the two-peak cross-flow VIV responses - which have been observed in some previous experiments with $m_{x} \neq m_{y}$ (Moe and Wu, 1990; Sarpkaya, $1995)$ - appear in Fig. 4a with $m^{*}{ }_{x}=m^{*}{ }_{y}$ whose $A_{y} / D$ values are as large as about 2.5. Nevertheless, the associated large values of $A_{x} / D$ are unrealistic.

Numerical results shown in Fig. 4 also show how both cross-flow and in-line VIV responses might disappear when specifying high and moderate values of $\varepsilon_{y}$, respectively. This is due to the fact that this empirical coefficient governs the system fluid damping regulating the self-limiting character of VIV response, see Eqs. (4) and (6). 


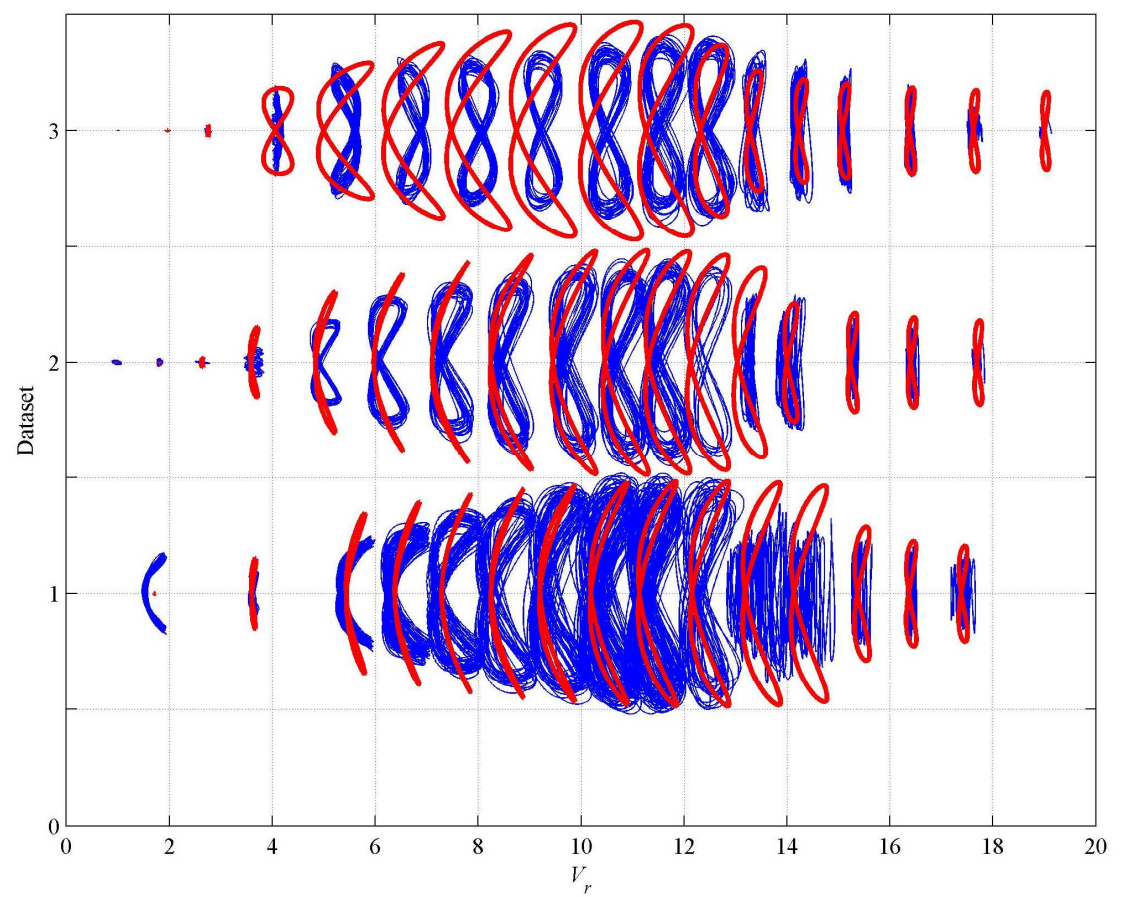

Figure 6 Experimental (blue lines) and numerical (red lines) comparisons of two-dimensional trajectories of in-line/cross-flow motions
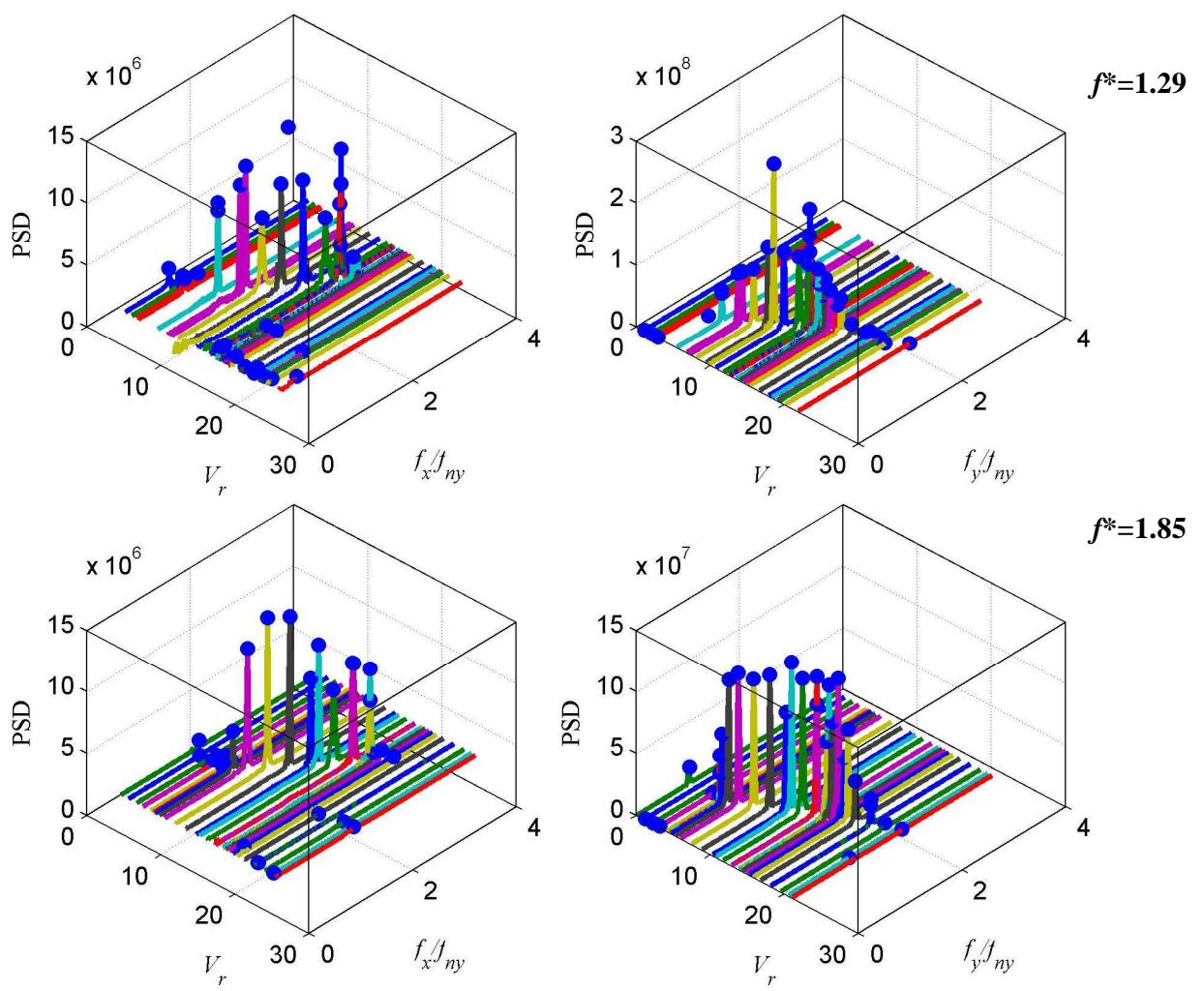

Figure 7 Inline and cross-flow frequency responses for experimental dataset $2\left(f^{*}=1.29\right)$ and $3(f *=1.85)$ 


\section{EXPERIMENTAL AND NUMERICAL COMPARISONS OF CROSS-FLOW AND IN-LINE VIV RESPONSES}

Experimental and numerical prediction results are now compared based on datasets in Table 1. As typical figure-ofeight orbital motions with tips pointing downstream (e.g. Fig. 2e) are mostly observed in the present experiments, the system equations of motions (Eqs. 3-6) used in numerical simulations are based on the model configuration shown in Fig. 2d. Both maximum and root-mean-squared (RMS) values of cross-flow $\left(A_{y} / \mathrm{D}\right)$ and in-line $\left(A_{x} / D\right)$ amplitudes are evaluated.

Results plotted in Fig. 5 illustrate a fairly good qualitative comparison of numerical and experimental responses. From the experiments, pure in-line responses are observed in a marginal range of about $2<V_{r}<4$ (Figs. 5b and d) whereas coexisting cross-flow/in-line VIV responses take place in the range of about $4<V_{r}<17.5$, depending on $f^{*}$. With increasing $V_{r}$, some clear jumps of peak amplitudes from upper to lower branches are experimentally (Figs. 5a and f) and numerically (Figs. 5a and b) observed. These jumps are in agreement with recently published experimental results of 2-DOF VIV with $f^{*}=1$ (Blevins and Coughran, 2009; Jauvtis and Williamson, 2004).

In view of quantitative comparisons, the highest values of experimental and numerical RMS amplitudes are found to be in the range of about $0.5-1.25$ for $A_{y} / D$ and $0.2-0.5$ for $A_{x} / D$, depending on the variable mass-damping value. Discrepancies increase in the comparison of maximum attainable or peak responses, except for $A_{x} / D$ in Fig. 5d. These outcomes could be influenced by the temporal modulation of $A_{y} / D$ and $A_{x} / D$ observed experimentally. With increasing $f^{*}$, some VIV behaviors are noticed experimentally. First, the in-line-only responses seem to disappear with $f^{*}=1.85$ (Fig. 5f). This is in agreement with the numerical prediction. Secondly, both crossflow and in-line responses in Figs. 5e and $\mathrm{f}\left(f^{*}=1.85\right)$ reveal the flattening slopes of their upper branches with amplitudes starting from $V_{r} \approx 2.5$ and ending at $V_{r} \approx 12.5$.

Overall, experimental results show maximum $A_{y} / D \approx 1.5$ and maximum $A_{x} / D \approx 0.5-0.8$, and the associated excitation ranges are quite comparable, in all $f^{*}$ cases. Given the similar values of $m^{*} \xi$, these imply the negligible effect of varying $f^{*}$ on the maximum response outcomes based on this pendulumspring-cylinder system. With respect to numerical comparisons, the predicted $A_{y} / D$ and $A_{x} / D$ are found to be overestimated and the associated upper branches show higher slopes being typical for resonance diagrams. These reflect the difficulty in matching numerical and experimental results in which several coefficients control the dynamic responses and some of the influential parameters are variable, i.e. $\xi_{x} \neq \xi_{y}$.

Next, it is of practical interest to perform numerical and experimental comparisons of the time-varying orbital $x-y$ motions as these could shed some light on how the cylinder oscillation affects the resulting vortex-shedding wake modes. Corresponding to results in Fig. 5, the $x$ - $y$ trajectory plots within several cycles of the oscillation are displayed in Fig. 6 with some chosen $V_{r}$.
Depending on $f^{*}, \xi$ (Table 1) and initial conditions in both numerical simulations and experiments, various characteristics of figure-of-eight trajectories appear with variable phase differences between $x$ and $y$ motions. In particular, the crescent shapes are evidenced in the experiments (see blue lines in Fig. 6) with their tips pointing mostly downstream (all datasets) and occasionally upstream (dataset 3 for $V_{r}<10$ ). The former case justifies the use of system equations based on the model configuration in Fig. 2d. Similar orbital motions have been found in recent 2-DOF VIV experiments of rigid circular cylinders (Blevins and Coughran, 2009; Dahl et al., 2006; Dahl et al., 2010; Jauvtis and Williamson, 2004), and the present study confirms these studies with both experimental and numerical results.

Figure 7 exemplified the oscillating frequency responses of $x$ and $y$ motions whose frequencies $f_{x}$ and $f_{y}$ are normalized by the cross-flow natural frequency $f_{n y}$, based on datasets 2 and 3 . It can be seen that, regardless of the specified $f^{*}$, the in-line and cross-flow oscillating frequencies mostly exhibit their nearlytuned 2:1 ratios: these correspond to the figure-of-eight appearance as a result of dual resonance shown in Fig. 6 . The frequencies contain single harmonics and reveal the negligible effect of higher-order terms.

It is worth noting that experimental orbital motions exhibit a high modulation feature of amplitudes whereby the oscillating cylinder does not follow the same path from cycle to cycle. This suggests a strong fluid-structure interaction effect during the test. On the contrary, numerical orbital motions are perfectly repeatable which justify the limit cycle character of the two pairs of coupled Duffing and van der Pol oscillators for which stable periodic solutions are attained. The numerical model is found to predict reasonably well several qualitative behaviors of the figure-eight appearance due to the associated quadratic nonlinearities (Srinil and Zanganeh, 2012).

\section{CONCLUSIONS}

Experimental studies on 2-DOF VIV of a flexibly mounted circular cylinder with a low equivalent mass ratio $\left(m^{*}=1.4\right)$ and variable in-line-to-cross-flow natural frequency ratio $\left(f^{*} \approx 1\right.$, $1.3,1.9)$ have been performed in a water towing tank. The flowinduced vibration experiments cover a sub-critical Re range of about 2000 - 50000. A generalized numerical prediction model has also been investigated based on double Duffing-van der Pol (structure-wake) oscillators which can capture the structurally geometrical coupling and fluid-structure interaction effects through system cubic and quadratic nonlinearities. The model empirical coefficients have been calibrated based on available experimental results and parametric investigations.

With $m^{*}$ being equal in both directions, the VIV excitation ranges have been experimentally found to be in a broad range of the reduced velocity parameter, $4<V_{r}<17.5$, with maximum attainable cross-flow and in-line amplitudes achieving high values of about 1.25-1.6 and 0.5-0.8, respectively, depending on the level and combination of the $x-y$ structural damping ratios in 
all $f^{*}$ cases. This damping parameter along with the twodirectionally geometrical coupling coefficients might in part be responsible for the disparity of response amplitudes and the quantitative differences between experimental and numerical results. Regardless of the specified $f^{*}$, figure-of-eight orbital motions have been experimentally as well as numerically observed throughout a large parametric range of $V_{r}$. These evidence the fundamental characteristics of dual 2:1 resonances of in-line and cross-flow responses in connection with the $2: 1$ forcing frequencies of the fluctuating drag/lift vortex-shedding forces.

\section{ACKNOWLEDGEMENTS}

The authors are grateful to the scholarships provided by the University of Strathclyde and the Scottish Funding Council through the Scottish Overseas Research Students Awards Scheme (SORSAS).

\section{REFERENCES}

Bearman, P.W., 2011. Circular cylinder wakes and vortexinduced vibrations. Journal of Fluids and Structures 27, 648658.

Blevins, R.D., 1990. Flow-Induced Vibrations. Van Nostrand Reinhold, New York.

Blevins, R.D., Coughran, C.S., 2009. Experimental investigation of vortex-induced vibration in one and two dimensions with variable mass, damping, and Reynolds number. Journal of Fluids Engineering 131, 101202-101207.

Currie, I.G., Turnbull, D.H., 1987. Streamwise oscillations of cylinders near the critical Reynolds number. Journal of Fluids and Structures 1, 185-196.

Dahl, J.M., Hover, F.S., Triantafyllou, M.S., 2006. Twodegree-of-freedom vortex-induced vibrations using a force assisted apparatus. Journal of Fluids and Structures 22, 807818.

Dahl, J.M., Hover, F.S., Triantafyllou, M.S., Oakley, O.H., 2010. Dual resonance in vortex-induced vibrations at subcritical and supercritical Reynolds numbers, Journal of Fluid Mechanics, pp. 395-424.

Facchinetti, M.L., de Langre, E., Biolley, F., 2004. Coupling of structure and wake oscillators in vortex-induced vibrations. Journal of Fluids and Structures 19, 123-140.

Gabbai, R.D., Benaroya, H., 2005. An overview of modeling and experiments of vortex-induced vibration of circular cylinders. Journal of Sound and Vibration 282, 575616.

Jauvtis, N., Williamson, C.H.K., 2004. The effect of two degrees of freedom on vortex-induced vibration at low mass and damping. Journal of Fluid Mechanics 509, 23-62.

Khalak, A., Williamson, C.H.K., 1999. Motions, forces and mode transitions in vortex-induced vibrations at low massdamping. Journal of Fluids and Structures 13, 813-851.

Moe, G., Wu, Z.J., 1990. The lift force on a cylinder vibrating in a current. Journal of Offshore Mechanics and Arctic Engineering 112, 297-303.
Nayfeh, A.H., 1993. Introduction to perturbation techniques. Wiley, New York.

Sarpkaya, T., 1995. Hydrodynamic damping, flow-induced oscillations, and biharmonic response. Journal of Offshore Mechanics and Arctic Engineering 117, 232-238.

Sarpkaya, T., 2004. A critical review of the intrinsic nature of vortex-induced vibrations. Journal of Fluids and Structures 19, 389-447.

Srinil, N., 2010. Multi-mode interactions in vortex-induced vibrations of flexible curved/straight structures with geometric nonlinearities. Journal of Fluids and Structures 26, 1098-1122.

Srinil, N., 2011. Analysis and prediction of vortex-induced vibrations of variable-tension vertical risers in linearly sheared currents. Applied Ocean Research 33, 41-53.

Srinil, N., Rega, G., 2007. Two-to-one resonant multimodal dynamics of horizontal/inclined cables. Part II: Internal resonance activation, reduced-order models and nonlinear normal modes. Nonlinear Dynamics 48, 253-274.

Srinil, N., Rega, G., Chucheepsakul, S., 2007. Two-to-one resonant multi-modal dynamics of horizontal/inclined cables. Part I: Theoretical formulation and model validation. Nonlinear Dynamics 48, 231-252.

Srinil, N., Wiercigroch, M., O’Brien, P., 2009. Reducedorder modelling of vortex-induced vibration of catenary riser. Ocean Engineering 36, 1404-1414.

Srinil, N., Zanganeh, H., 2012. Modelling of coupled cross-flow/in-line vortex-induced vibrations using double Duffing and van der Pol oscillators. Ocean Engineering 53, 8397.

Stappenbelt, B., Lalji, F., Tan, G., 2007. Low mass ratio vortex-induced motion, The 16th Australasian Fluid Mechanics Conference, Gold Coast, Australia,, pp. 1491-1497.

Sumer, B.M., Fredsoe, J., 2006. Hydrodynamics Around Cylindrical Structures. World Scientific.

Trim, A.D., Braaten, H., Lie, H., Tognarelli, M.A., 2005. Experimental investigation of vortex-induced vibration of long marine risers. Journal of Fluids and Structures 21, 335-361.

Vandiver, J.K., Jong, J.Y., 1987. The relationship between in-line and cross-flow vortex-induced vibration of cylinders Journal of Fluids and Structures 1, 381-399.

Wang, X.Q., So, R.M.C., Chan, K.T., 2003. A non-linear fluid force model for vortex-induced vibration of an elastic cylinder. Journal of Sound and Vibration 260, 287-305.

Williamson, C.H.K., Govardhan, R., 2004. Vortex-induced vibrations. Annual Review of Fluid Mechanics 36, 413-455.

Williamson, C.H.K., Jauvtis, N., 2004. A high-amplitude $2 \mathrm{~T}$ mode of vortex-induced vibration for a light body in $\mathrm{XY}$ motion. European Journal of Mechanics - B/Fluids 23, 107-114.

Zanganeh, H., Srinil, N., 2012. Interaction of wake and structure in two-dimensional vortex-induced vibrations, International Conference on Advances and Challenges in Marine Noise and Vibration, Glasgow, pp. 177-188. 